\title{
Editorial zum Schwerpunkt: Freiheit und Gesellschaft
}

Heft 2/2003 widmet sich im Schwerpunkt dem Spannungsverhältnis von individueller Freiheit und Gesellschaft. Dieses spiegelt sich nicht nur in der aktuellen Sicherheitsdebatte (Peter-Alexis Albrecht) oder in der Kontrollunterwerfung einer wenig aufgeklärten Öffentlichkeit (Stefan Braum) wider, sondern ist auch Kennzeichen weiterer Problemlagen des modernen Rechts. Dabei muss der Blick immer wieder auf die Funktion der Grundrechte fallen. Institutsgarantien (Sandra Obermeyer), der Bezug zu einer angeblich objektiven Werteordnung und ähnliche Verformungen eines in der Verfassung prinzipiell angelegten individuellen Grundrechtsschutzes im Sinne von Abwehrrechten kulminieren in einem »Grundrecht auf Sicherheit « - eine Art verfassungsrechtliches Totschlagsargument zu Lasten des individuellen Freiheitsschutzes. Die Gefährdung der Autonomie begleitet den Menschen offenbar »von der Wiege bis zur Bahre«. Insofern sind die Themen Sterbehilfe (Lars Witteck) und Lebensschutz (Erhard Denninger) - sogar schon vor Nidation und Geburt - Eckpunkte für das Spannungsfeld grundsätzlicher Debatten über menschliche Autonomie und Würde. Das Ziel der wissenschaftlichen Arbeit unseres Strafrechtskollegen Alessandro Baratta, der am 25. Mai 2002 verstorben ist, war es, die Grundpfeiler einer Politik der Verteidigung der Grundrechte und der Befriedigung der menschlichen Bedürfnisse herauszuarbeiten. In einem solchen System soll das Strafrecht einen subsidiären Stellenwert haben, in Barattas Worten: Ein minimales Strafrecht in den Grenzen der Verfassung. Einer seiner letzten Beiträge zielt in die Mitte der Grundrechtsproblematik, der Freiheit und der Menschenwürde: In dem Beitrag Kriminalpolitik und Verfassung lässt Baratta fundamentale Überlegungen zum minimalen Strafrecht und zur Sicherheit der Rechte aufscheinen. Er ruft auf zur Eröffnung »interdisziplinärer Werkstätten..., die dem Problem der Sicherheit der Rechte gewidmet sein werden«. Alle Beiträge dieses Heftes verstehen sich in diesem Baratta'schen Sinne als Bestandteile des Verfassungsprojekts einer Politik der Grundrechte.

Ausgangspunkt für die zentrale Fragestellung ist, ob gesellschaftliche Bedrohungslagen - scheinbare oder reale - es rechtfertigen, die Kernfunktion der Grundrechte, nämlich den Schutz vor staatlichem Freiheitsverzehr zu gewähren, langsam aufgehen lassen in schützender Fürsorge für und durch den Staat. Auftakt hierfür bildet in diesem Heft ein Briefwechsel, der durch den Vortrag »Die vergessene Freiheit « auf einer Tagung der Friedrich-Ebert-Stiftung in Berlin im März 2003 den Wissenschaftskollegen Fritz Sack $\mathrm{zu}$ heftiger freundschaftlicher Reaktion (Lieber Alexis) veranlaßte. Ein Restatement (Lieber Fritz) - ebenso heftig freundschaftlich - durfte nicht ausbleiben. Das editorische Motiv, der Leserschaft diese persönlichen Reaktionen zuzumuten, will Neugier für die Lektüre wecken und kenntlich machen, daß Positionen der Aufklärung und der Einsatz für die Freiheit nicht antiquierte historische Relikte, sondern konkrete Leitlinien für den Umgang mit »modernen« Herausforderungen für Staat und Gesellschaft sein können. Die Entscheidung für den Abdruck trafen die Autoren erst nach dem Briefwechsel: 
Lieber Alexis,

ich muss doch noch ein »Nachwort « zur Tagung der Friedrich-Ebert-Stiftung los werden. Ich habe noch heftig darüber nachgedacht, insbesondere über die abschliessende Podiumsdiskussion, die mir irgendwie gespenstisch vorkam.

Nun war dies ja eine Veranstaltung von zwei Akteuren im politisch-ideologischen Feld und deshalb überkam mich zunehmend ein Unbehagen, das ich auch wachsend im Bundesvorstand der Humanistischen Union verspüre: eine Resolution nach der anderen gegen den Verfall von Rechtsstaat, Freiheit etc. geht den Bach herunterHirsch brachte es ja in das plastische Bild: der Rechtsstaat - von der schiefen Bahn zum freien Fall in den totalitären Überwachungsstaat. Du hast den Ton der »Rechtgläubigen « ja wohl am besten getroffen - die meiste Resonanz gekriegt. Ich bin mit meinen Überlegungen nicht so recht angekommen - zum Teil lag es wohl an der Vortragsart, wohl aber auch daran, dass ich mich nicht so lange mit dem Klagelied selbst aufgehalten habe.

Erlaube mir ein paar Nachfragen wie Feststellungen:

1. Kann es sein, dass die Heftigkeit deiner Wortwahl und die disziplinierte Aufgeregtheit deines Vortrags nicht nur Ausdruck der vorgeführten Diskrepanz zwischen Verfassungsnorm und Verfassungswirklichkeit, sondern auch einer uneingestandenen Erfahrung über die Ausweglosigkeit des von dir gewählten Pfades ist? Wann gehst du in den Untergrund? - so war ich unterwegs geneigt, mich zu fragen!

2. Auf dem Weg von Berlin nach Hamburg kam mir der Gedanke, dass deine Position sowie diejenige einiger anderer Referenten und der meisten Zuhörer eigentlich dem Motto entsprach, das ich von dem früheren englischen Premierminister John Major zitiert habe - nur halt Seiten verkehrt: »condemn more, understand less «es wurde kräftigst auf all die Rechtsstaatsverächter und-zerstörer gewettert und nur wenig Bereitschaft gezeigt, der ja nicht bestrittenen Diagnose auch »verstehend « zu Leibe zu rücken. Das ist natürlich Lagerverhalten - aber du bist doch Wissenschaftler, dazu noch jemand aus dem sozialwissenschaftlichen Bereich!

3. Zwischendurch fühlte ich mich wie auf einer Veranstaltung, auf der der »Untergang des Abendlandes « "gemenetekelt« wird - ich habe zwar eine solche Veranstaltung noch nie erlebt, aber so beklemmend kam es mir streckenweise vor.

4. Ebenfalls kenne ich es nur als Parodie aus der Ferne und vom »Klassengegner", als man zu Zeiten noch der beiden deutschen Staaten die Haltung der Inhaber der DDR-Macht so karikierte, dass man ihnen zwecks Erhaltung und Verlängerung ihrer Existenz die Wahl eines neuen Volkes empfahl. Das Ausmass und die Bereitschaft, sich selbst »Rationalität«, »Vernunft«, den »Durchblick« auf die Situation und auf den Gang der Dinge zu attestieren und den Gegner ins irrationale, unverantwortliche und inkompetente Abseits zu stellen, empfand ich von einer Selbstgerechtigkeit und mangelnden Skepsis gekennzeichnet, die so nicht richtig und angemessen sein kann. Auf die Spitze getrieben, wozu man gar keine grosse Fantasie benötigte, bedeutet es genau die Konsequenz, die man einst aus der »Entfremdung « der sozialistischen Führung von ihrem sozialistischen Volk satirisch gezogen 
hat. Insbesondere bei der Podiumsdiskussion hatte ich das Gefühl: wir hier drinnen die Rechtgläubigen vs. die da draussen die Abtrünnigen und Fahnenflüchtigen. Ich habe mich gefragt, warum unsere beiden »Parlamentarier « Herta Däubler-Gmelin und Burkhard Hirsch, die sich ja so einig in ihrer Klage um den Verfall des Rechtsstaats gaben, nicht einen Gedanken darüber äusserten, warum es ihre Parlamentskollegen ihnen denn nicht gleich tun - das drängte sich doch geradezu auf!

5. Eine allerletzte Analogie, die mir kam: als wir noch in den sechziger Jahren waren, hat man den Seminarmarxisten vorgehalten, dass sie in monotoner Einfallslosigkeit immer weiter nur die exegetische Aneignung der Marxschen Schriften betrieben, immer weiter machten, selbst als ihnen die Zuhörer und das Publikum weg blieben und sie am Ende nur noch autistische Selbstgespräche führten. Sind wir Gralshüter von Rechtsstaat und Freiheit nicht auch in dieser Gefahr? Auch wir registrieren die abnehmende Resonanz und Attraktivität unserer Botschaften - und reagieren darauf, diese immer lauter und schriller zu verkünden: irgendwie ist dieser Zirkel autodestruktiv bis zu seiner »Implosion«.

6. Meine Antwort darauf ist: Innehalten, Nachdenken, neue »Feindbestimmung «ersuchen. Ich finde Garlands resumé sehr bedenkenswert: »From protection from the state to protection by the state «: wir sind mit einer Neubestimmung des Verhältnisses von Staat zu Gesellschaft konfrontiert.

Grüsse von Fritz.

\section{Lieber Fritz,}

ich bin nicht im Untergrund, sondern oben, auf den Bergen. Von dort schaut es sich gut nach unten. Dein 6-Punkte-Programm krankt meines Erachtens an einer zentralen Stelle. Du meinst, die Diagnose sei nicht bestritten, was ich aufs äußerste bestreite. Es gibt aus meiner Sicht keine gesellschaftliche Lage, die einen Ausnahmezustand für ein nicht-rechtsstaatliches Strafrecht rechtfertigt. Es gibt dafür einen Haufen rechtspolitischer Szenarien, mittels derer eine hilflose Politik sich im Sinne eines Kommunikationsmediums für allfällige Wahlen positioniert. Verschärftes Strafrecht - und das steht für Gebrüll gegen Verunsicherung, Angst vor notwendigen Strukturveränderungen etc. - soll die Wunderwaffe sein, mittels derer eine unfähige Bande von Verantwortungsträgern Zukunftsmanagement vorzugeben trachtet - um gewählt zu werden. Die gesellschaftlichen Problemzusammenhänge transparent zu machen und strukturpolitische Lösungsvorschläge zu unterbreiten ist offenbar für Politiker unerreichbares Ziel. Deshalb dient das Strafrecht als Kommunikationsmedium, und zwar international: Härter, schärfer, schneller, konsequenter! Alles Parolen tumber Unfähigkeit.

Unfähig ist die (Rechts-)Politik, die die Realitäten zum großen Teil nicht wahrnimmt oder diese - häufig wider besseres Wissen - nicht vermittelt. Warum nicht? Weil die Nähe zur Macht ganz offenbar das Bewußtsein vernebelt und die Angst vor dem Verlust von Einfluß und Macht immer nur auf Nummer sicher setzen läßt, das heißt auf aktuelle Stimmungen und Meinungsmache. Demokratie verkommt zur Demoskopie! Aufklärung gehört an deren Stelle, zum Beispiel wie folgt: Es gibt seit hundert Jahren keine statistisch nachweisbare Steigerung von Kriminalitätsverarbeitung, stets - bis 
auf die Zeit des Dritten Reiches - verurteilte und verurteilt die deutsche Justiz weniger als ein Prozent der Bevölkerung per anno - inklusive Straßenverkehrsdelikte und aller Bagatellen. Steigerungen in der polizeilichen Verdachtsstatistik werden informell und als »Fallast « erledigt: leider zumeist ohne Justizförmigkeit und unter erheblicher Grundrechteeinschränkung. Kriminalität bedroht die Grundfesten dieser Gesellschaft in keiner Weise: Terrorismus, Organisierte Kriminalität, Ausländerkriminalität sind primär Horrorszenarien, die die Medien und die Interessenpolitiken wohlfeil pflegen. Bei näherem, das heißt wissenschaftlichem Hinsehen: empirische Fehlanzeige! Das darf man nicht nur sagen, das muß man sagen, klar und deutlich, immer wieder.

In geschürter Panik wird der Rechtsstaat, also die Grundprinzipien dieser verfaßten Gesellschaft, das ist Gesetzlichkeit, Legalität, individuelle Verantwortlichkeit (auch als Zuschreibung!), Verhältnismäßigkeit, staatliche Zuständigkeiten und Fairneß, über den Haufen geschmissen: Das gehört eigentlich bestraft! Insofern bin ich gerne ein Gralshüter von Rechtsstaat und Freiheit. Mein Schaffen für ein Strafrecht der Strafgesetzlichkeit kann ich nicht deshalb aufgeben, weil eine politische Narrenzunft andere Interessen mit einem populistischen Strafrecht verfolgt, ich singe das Lied weiter. Ein freiheitliches Kernstrafrecht kann und muß Unrecht, das den Bürgern widerfährt, kenntlich machen! Mehr kann es nicht, aber das muß es! Es muß dafür eng, präzise, gesetzesgebunden, freiheitssichernd sein, vor allem - mit aktuellem Blick muß es Bestandteil europäischer Verfassung werden.

Natürlich gibt es gesellschaftliche Risikolagen, die unsere Existenz bedrohen. Das sind makrosoziale Gefahren, die aus ungezügelter Macht, staatlichem Omnipotenzstreben und egozentrischen Wirtschaftspolitiken entspringen. Möglicherweise sind hier die Ursachen punitiver Tendenzen in der Gesellschaft zu sehen, quasi als destruktive Ohnmachtsreaktionen. Nur: Hierfür gibt das Strafrecht keine Antworten und Hilfen, nur Scheinantworten: Derartige Systemschutz-Anforderungen zerbrechen das rechtsstaatliche Strafrecht. Die symbolischen Anforderungen mißbrauchen es. Alle derartigen Überforderungen zerstören ein jedes rechtsstaatliches Kriminaljustizsystem. Meine Erwartungen an ein Kriminaljustizsystem der Strafgesetzlichkeit sind eindeutig und bescheiden zugleich: Faires Verfahren und rechtsstaatliche Vorbildlichkeit sind die Topoi, die den Rechtsstaat unschlagbar machen, gerade in Zeiten der Gefahr. Ich suche mir dafür kein neues Volk, man kann nur versuchen zu überzeugen. Das ist aus meiner Sicht auch unsere Aufgabe als Wissenschaftler.

Grüsse von Alexis

Die Herausgeber der Kritischen Vierteljahresschrift für Gesetzgebung und Rechtswissenschaft widmen diesen Schwerpunkt dem Gedenken unseres Kollegen Alessandro Baratta, dem der Einsatz für die Menschenrechte und die sie konkretisierenden Grundrechte lebenslanges wissenschaftliches Anliegen war. Mit der Entwicklung eines verfassten »minimalen « Kriminalstrafrechts, das Freiheit - und damit menschliche Autonomie und Würde - schützen will, wird sein wissenschaftliches Werk und sein Name für immer verbunden sein. 